\title{
Modeling of Electron Tunneling Current in a p-n Junction Based on Strained Armchair Graphene Nanoribbon
}

\author{
Rifky Syariati, Endi Suhendi, Fatimah A. Noor, Mikrajuddin Abdullah, and Khairurrijal
}

\begin{abstract}
A theoretical model of electron tunneling current in a p-n junction made from strained armchair graphenenanoribbon (AGNR) was developed. The effect of strain to the energy dispersion relation of AGNR was formulated under the tight binding method. The energy bandgap of AGNR was then analytically determined. Furthermore, the electron transmittance was calculated by utilizing the Airy wavefunction-approach. Finally, the calculated transmittance was used to obtain the tunneling current by employing the Landauer formula. The effects of strain to the energy band gap, AGNR width, and tunneling current were studied thoroughly.
\end{abstract}

Index Terms - Graphene, AGNR, tight binding, strain, band gap, p-n junction, Airy wave function, tunneling current.

\section{INTRODUCTION}

Graphene has recently attracted considerable as a potential candidate material for nanoelectronics due to its electronics properties. Basically, graphene is a single-atom-thick material composed of carbon atoms forming a hexagonal lattice. When the width is smaller than the length, graphene becomes graphenenanoribbons (GNRs) which can be metallic or semiconducting depending on their structures [1]-[4]. Armchair graphenenanoribbon (AGNR) is one type of GNRs which has semiconductor properties [5]. Because of the properties, AGNRs can be applied for various electronic devices including p-n junction diodes [6], [7].

One of the important electronic properties of GNRs is energy bandgap. The bandgap of GNRs can be changed by manipulating their geometry and width. Beside those, the band gap of GNRs can be altered by giving a strain to the lattice of GNR. The strained lattice of GNR will affect the form and width of GNR to result in the change in the bandgap. Tight binding method is one of the methods in obtaining the bandgap of GNRs that depends on the lattice structure and atomic position [8], [9]

In this paper, we report the studies on the strained AGNR by using the tight binding method and its implication to the tunneling current through the strained potential barrier of AGNR in a p-n junction diode. The tunneling current was derived by employing the Airy wavefunction-approach and calculated by using the Landauer formula. The effects of strain to the energy bandgap, AGNR width, and tunneling current will be discussed in detail.

Manuscript received May 15, 2014; revised July 19, 2014.

Rifky Syariati, EndiSuhendi, Fatimah A. Noor, Mikrajuddin Abdullah, and Khairurrijal are with the Department of Physics, Institut Teknologi Bandung, Indonesia (e-mail: rifkyrifky89@yahoo.co.id, endisuhendi@yahoo.com, fatimah@fi.itb.ac.id, din@fi.itb.ac.id, krijal@fi.itb.ac.id).

\section{THEORETICAL MODEL}

Fig. 1 (a) and Fig. 1 (b) illustrate the lattice crystal and atomic position of GNR without and with applying a strain to the GNR, respectively. Under the tight binding model, the Hamiltonian and the energy dispersion relation is described by [8]

$$
\begin{gathered}
H=\left(\begin{array}{cc}
E_{\mathrm{o}} & t f(k) \\
t f^{*}(k) & E_{\mathrm{o}}
\end{array}\right) \\
E(k)=E_{\mathrm{o}} \pm t|f(k)|
\end{gathered}
$$

where $E_{0}$ is the initial energy of electron and $t$ is the overlapping electron parameter which has a magnitude of $2.76 \mathrm{eV}$ [8]. The geometry factor of GNR, $f(k)$, is defined by

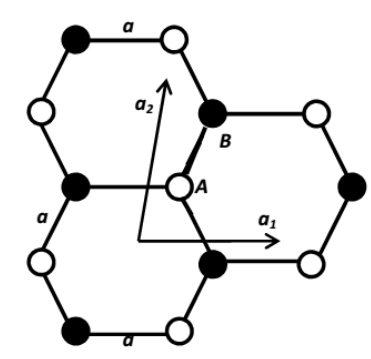

(a)

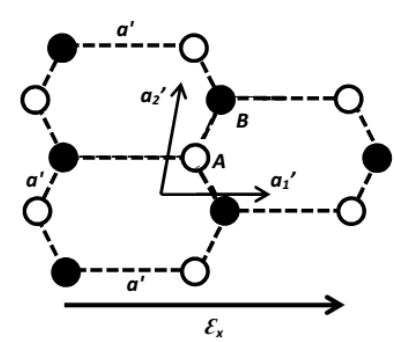

(b)
Fig. 1. The lattice structure of GNR with atoms A and B before (a) and after applying a strain (b).

$$
f(k)=\sum_{m_{1}}^{N} e^{-i k m_{1}},
$$

where $k$ is the wave number and $m$ is the atom position. When the strain was applied to the GNR, the positions of atoms become as follows [10]:

$$
\begin{gathered}
m_{1}=a\left(1+\varepsilon_{\mathrm{y}}\right) e_{\mathrm{y}}, \\
m_{2}=\frac{\sqrt{3}}{2} a\left(\left(1+\varepsilon_{\mathrm{x}}\right) e_{\mathrm{x}}-\frac{1}{\sqrt{3}}\left(1+\varepsilon_{\mathrm{y}}\right) e_{\mathrm{y}}\right), \\
m_{3}=\frac{\sqrt{3}}{2} a\left(-\left(1+\varepsilon_{\mathrm{x}}\right) e_{\mathrm{x}}-\frac{1}{\sqrt{3}}\left(1+\varepsilon_{\mathrm{y}}\right) e_{\mathrm{y}}\right),
\end{gathered}
$$

where $a$ is a distance between two carbon atoms, $\varepsilon_{x}$ is the strain on $\mathrm{x}$-axis and $\varepsilon_{y}$ is the strain on $\mathrm{y}$-axis. The relation between $\varepsilon_{x}$ and $\varepsilon_{y}$ is written by [11] 


$$
v=-\frac{\varepsilon_{y}}{\varepsilon_{x}},
$$

where $v$ is the Poisson ratio with the magnitude of 0.186 [10]. Furthermore, Eqs. (4), (5), and (6) are substituted into Eq. (3) and then substituted into Eq.(2), thus, we obtain

$$
\begin{aligned}
& f(k)=\sqrt{1+\cos ^{2}\left(\left(1+\varepsilon_{\mathrm{x}}\right) k_{\mathrm{x}} \frac{\sqrt{3}}{2} a\right)+4 \cos \left(\left(1+\varepsilon_{\mathrm{x}}\right) k_{\mathrm{x}} \frac{\sqrt{3}}{2} a\right) \cos \left(\left(1+\varepsilon_{\mathrm{y}}\right) k_{\mathrm{y}} \frac{3}{2} a\right)} . \\
& E=? \frac{3 a t}{2} \sqrt{+\cos ^{2}\left(\left(1+\varepsilon_{\mathrm{x}}\right) k_{\mathrm{x}} \frac{\sqrt{3}}{2} a\right)+4 \cos \left(\left(1+\varepsilon_{\mathrm{x}}\right) k_{\mathrm{x}} \frac{\sqrt{3}}{2} a\right) \cos \left(\left(1+\varepsilon_{\mathrm{y}}\right) k_{\mathrm{y}} \frac{3}{2} a\right)} .
\end{aligned}
$$

Here, the term of $(3 a t / 2)$ is equal to $\hbar v_{\mathrm{f}}$, where $v_{\mathrm{f}}$ is the Fermi velocity [8], [9].

The next step is how to find the electron characteristics in the places where the conduction and valence bands are in the low states. These locations are known as points $\mathrm{K}$ and $\mathrm{K}$ ' with slight shift $p$ or mathematically can be written by $k=K+p$ and $k=K^{\prime}+p$, where $K=\frac{4 \pi}{3 \sqrt{3} a} e_{x}$ and $K^{\prime}=$ $-\frac{4 \pi}{3 \sqrt{3} a} e_{x}[1]$, the geometry factor becomes

$$
f(p)=\frac{3 a}{2}\left(\left(1+\varepsilon_{x}\right) p_{x}-i\left(1+\varepsilon_{y}\right) p_{y}\right) .
$$

where $p_{x}$ and $p_{y}$ are wave number in $x$-axis and $y$-axis respectively.

In order to obtain the energy dispersion relation at the point $\mathrm{K}$ and $\mathrm{K}$ ', it is assumed that the initial energy electron is zero, and the energy becomes

$$
E= \pm \frac{3 a t}{2} \sqrt{\left(1+\varepsilon_{x}\right)^{2} p_{x}^{2}+\left(1+\varepsilon_{y}\right)^{2} p_{y}^{2}} .
$$

The symbol of \pm indicates the conduction band $(+)$ and valence band (-). The magnitude of $p_{\mathrm{x}}$ in AGNRs is determined by the boundary condition [10] which is given by

$$
p_{\mathrm{x}}=\frac{1}{\left(1+\varepsilon_{\mathrm{x}}\right)}\left[\frac{\pi n}{W_{\mathrm{ac}}+\sqrt{3} a}+\frac{4 \pi}{3 \sqrt{3} a}\right] \text {, }
$$

where $W_{\mathrm{ac}}$ is the width of AGNR.
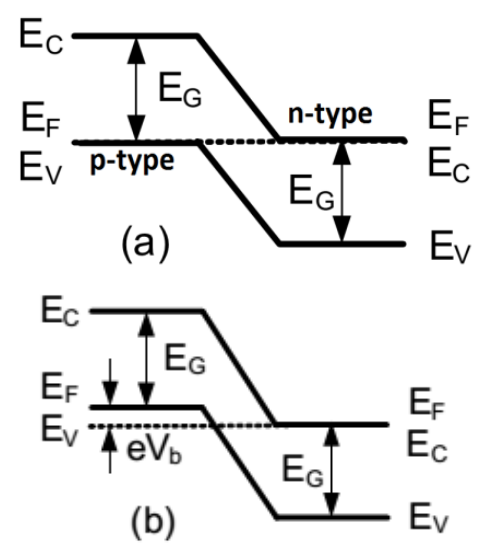

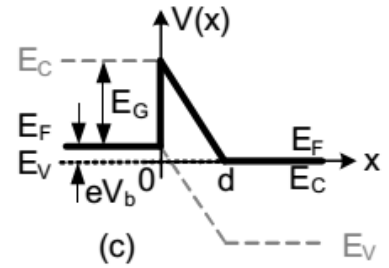

Fig. 2. (a) An energy band diagram of a p-n junction diode in thermal equilibrium, (b) when the reserve-bias voltage is applied to a p-n junction, and (c) a model of potential barrier.

Fig. 2 (a) illustrates the energy band diagram of a p-n junction based on AGNRs in the thermal equilibrium. When the reverse bias voltage is applied to the $p-n$ junction, the valence band of the $\mathrm{p}$ region is higher than the conduction band of the $n$ region (Fig. 1 (b) ). Then, electrons in the valence band of $\mathrm{p}$ region tunnel through the depletion region toward the conduction band of $\mathrm{n}$ region, which is known as Zener tunneling as given in Fig. 2 (c) [6], [7].

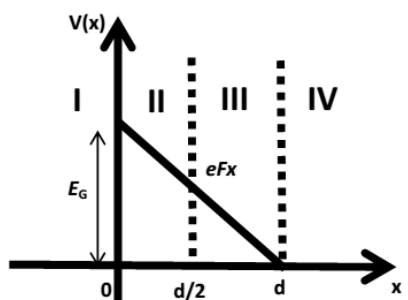

Fig. 3. Potential barrier when a small bias voltage is applied.

The electron transmittance was derived by using the Airy wavefunction-approach following the method in Ref. [7] but we solved it numerically. In the Fig. 3, the wave function in each region is defined by

$$
\begin{gathered}
\psi_{I}(x)=A \exp \left(i k_{1} x\right)+B \exp \left(-i k_{1} x\right), x<0, \\
\psi_{I I}(x)=C A i(\zeta(x))+D B i(\zeta(x)), 0 \leq x \leq \frac{d}{2}, \\
\psi_{I I I}(x)=E A i(\xi(x))+F B i(\xi(x)), \frac{d}{2} \leq x \leq d, \\
\psi_{I V}(x)=G \exp \left(i k_{2} x\right), x>d,
\end{gathered}
$$

where $A, B, C, D, E, F$, and $G$ are constant, $A i$ and $B i$ are Airy function. In Eq.(13) the wave number in the region $I$ is written as

$$
k_{1}=\sqrt{\frac{2 m_{1} E}{\hbar^{2}}},
$$

where $m_{1}$ is electron effective mass in $p$-junction, $E$ is electron energy and $\hbar$ is reduced Planck constant. In the region II and III the Airy function is expressed by

$$
\begin{aligned}
& \zeta(x)=\left(\frac{2 m_{1} e F}{\hbar^{2}}\right)^{\frac{1}{3}}\left(\frac{E_{G}-E}{e F}-x\right), \\
& \xi(x)=\left(\frac{2 m_{2} e F}{\hbar^{2}}\right)^{\frac{1}{3}}\left(\frac{E_{G}-E}{e F}-x\right),
\end{aligned}
$$


where $m_{2}$ is electron effective mass in $n$-junction, $E_{\mathrm{G}}$ is the band gap and $F$ is the applied electric field. And then for the region IV, the wave number is illustrated by

$$
k_{2}=\sqrt{\frac{2 m_{2} E}{\hbar^{2}}}
$$

Applying boundary conditions between two regions, the wave function becomes

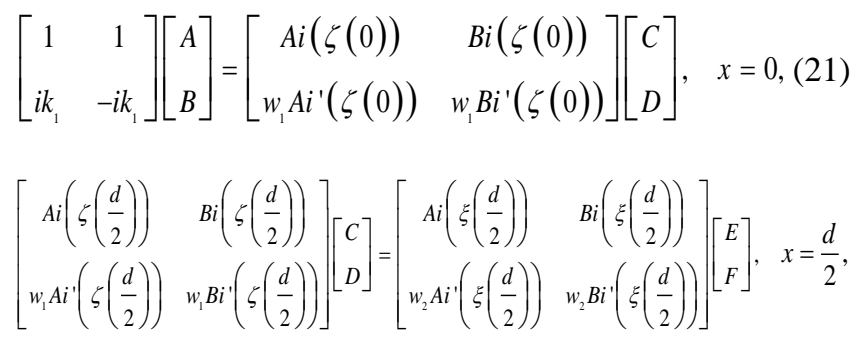

$\left[\begin{array}{cc}A i(\xi(d)) & B i(\xi(d)) \\ w_{2} A i^{\prime}(\xi(d)) & w_{2} B i^{\prime}(\xi(d))\end{array}\right]\left[\begin{array}{c}E \\ F\end{array}\right]=e^{i k_{2} d}\left[\begin{array}{c}G \\ i k_{2} G\end{array}\right], \quad x=d$.

where $w_{1}=\left(\frac{2 m_{1} e F}{\hbar^{2}}\right)^{\frac{1}{3}}$ and $w_{2}=\left(\frac{2 m_{2} e F}{\hbar^{2}}\right)^{\frac{1}{3}}$

From the equation (21), (22), and (23), we obtain the transmittance

$$
T=\frac{k_{2}}{k_{1}}\left(\frac{G}{A}\right)^{*}\left(\frac{G}{A}\right)
$$

The obtained transmittance was then employed to calculate the tunneling current by using Eq. (25).

$$
I=\frac{2 g_{\mathrm{v}} e}{h} \int_{0}^{e V_{\mathrm{b}}}\left[f_{\mathrm{v}}(E)-f_{\mathrm{c}}(E)\right] T d E
$$

where $\quad f_{\mathrm{v}}(E)=1 /\left(1+\exp \left[\left(E-e V_{\mathrm{b}}\right) / k_{\mathrm{b}} T\right]\right) \quad$ and $f_{\mathrm{c}}(E)=1 /\left(1+\exp \left[E / k_{\mathrm{b}} T\right]\right)$ are the Fermi-Dirac distributions in the valence and the conduction bands, respectively, $k_{\mathrm{b}}$ is Boltzmann constant, $g_{\mathrm{v}}$ is GNR degeneration which has a magnitude of 1 , and $\hbar$ is the Planck constant.

\section{CAlCUlated Results AND Discussions}

Fig. 4 (a) shows the strain effect to the energy dispersion relationship and the energy bandgap, respectively. The AGNR width was taken as $10 \mathrm{~nm}$. It is shown that the energy dispersion relationship between the conduction and valence band edges is changed when the strain was applied as seen in Fig. 4 (a). It is also shown that the energy bandgap gets wider with the strain given to the AGNR as depicted in Fig. 4 (b). It could be explained as follows: when the strain force was applied to the AGNR, the atoms of honey comb lattice will repel each other so that the electrons require a greater energy to move to another position. On the other hand, it was known that the AGNRs band gap is strongly influenced by the width [8], [9] so that if the width is changed by the strain, the band gap will also change. These results show that the strain gives an effect to the energy dispersion and band gap of AGNR.

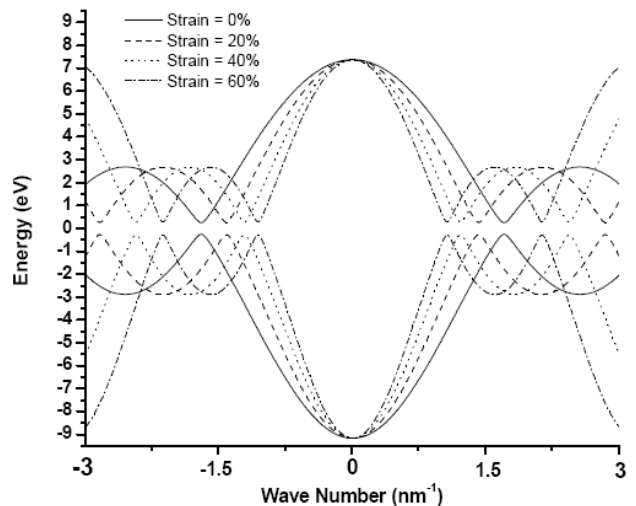

(a)

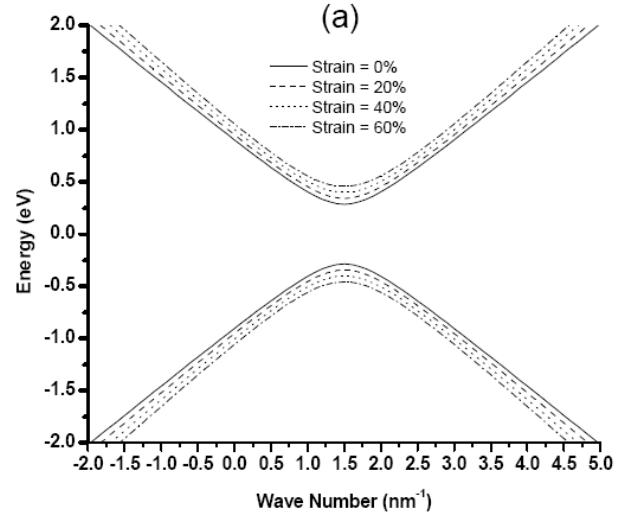

(b)

Fig. 4. (a) The effect of strain to the energy dispersion relationship and (b) The change of the energy band gap for various strains.

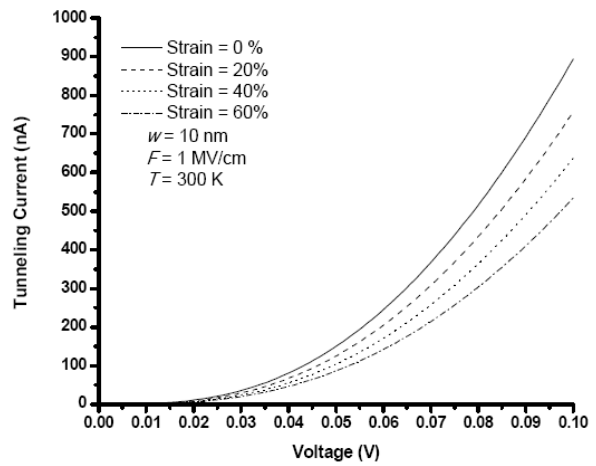

a)

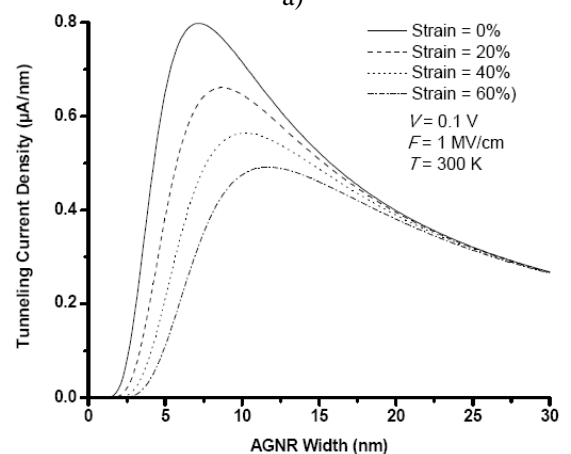

b)

Fig. 5. (a) I-V characteristics of an AGNR-based p-n junction for various strains and (b)Tunneling current density as a function of AGNR width for various strains. 
Fig. 5 (a) depicts the tunneling current versus bias voltage for various strains. The AGNR width, electric field, and temperature were $10 \mathrm{~nm}, 1 \mathrm{MV} / \mathrm{cm}$, and $300 \mathrm{~K}$, respectively. It is shown that the tunneling current increases as the strain decreases and it reaches the highest when without giving a strain to the AGNR. It happens because the band gap becomes narrow as the strain decreases as given in Fig. 4 (b). The potential barrier was then lowered so that the electrons become easier to tunnel through the barrier resulting in the increase in the tunneling current. The strain effect to the tunneling current and width of AGNR is shown in Fig. 5 (b). The parameters $V=0.1 \mathrm{~V}, F=1 \mathrm{MV} / \mathrm{cm}$, and $T=300 \mathrm{~K}$ were taken. It is seen that the tunneling current increases with decreasing the strain effect and width. It is also observed that the strain does not affect to the tunneling current for the AGNR width thicker than $20 \mathrm{~nm}$.

\section{CONCLUSION}

We have studied the strain effect to an AGNR-based p-n junction diode by using the tight binding method. It has been shown that the strain effect cannot be neglected in determining the dispersion energy relation and band gap. It has been also observed that the tunneling current increases with decreasing the strain effect. However, the strain does not affect to the tunneling current for thicker ribbon width of AGNR.

\section{ACKNOWLEDGMENT}

This work was financially supported by "HibahDesentralisasi", "Riset \& Inovasi KK", and "HibahKompetensi" Research Grants in the fiscal year 2013-2014.

\section{REFERENCES}

[1] D. Jiménez, “A current-voltage model for Schottky-barrier graphene-based transistors," Nanotechnology, vol. 9, no. 34, p. 345204 , July 2008.

[2] J. Fernandez-Rossier, J. J. Palacios, and L. Brey, "Electronic structure of gated graphene and graphene ribbons," Phys. Rev. B, vol. 75, p. 205441, May 2007.

[3] N. M. R. Peres, A. H. Castro Neto, and F. Guinea, "Conductance quantization in mesoscopicgraphene," Phys. Rev. B, vol. 73, p. 195411, May 2006

[4] F. Munoz-Rojas, D. Jacob, J. Fernandez-Rossier, and J. J. Palacios, "Coherent transport in graphenenanoconstrictions," Phys. Rev. B, vol. 74, p. 19541, Nov. 2006.

[5] H. Zheng, Z. F. Wang, T. Luo, O. W. Shi, and J. Chen, "Analytical study of electronic structure in armchair graphenenanoribbons," Phys. Rev. B, vol. 75, p. 165414, April 2007.

[6] D. Jena, T. Fang, Q. Zhang, and H. Xing, "Zener tunneling in semiconducting nanotube and graphenenanoribbon p-n junctions," Appl. Phys. Lett., vol. 93, p.112106, Sep. 2008.

[7] W. Yansen, M. Abdullah, and Khairurrijal, "Application of airy function approach to model electron tunneling in graphenenanoribbon-based p-n junction diodes," Jurnal Nanosains \& Nanoteknologi, vol. 3, no. 1, pp. 18-21, Feb. 2010.

[8] C. Neto, F. Guinea, R. Peres, S. Novoselov, and K. Geim, "The electronic properties of graphene," Rev. Mod. Phys., vol. 81. p. 109, Jan. 2009.

[9] A. K. Geim and K. S. Novoselov, "The rise of graphene," Nat. Mater., vol. 6, pp. 183-191, Mar. 2007.

[10] Y. Li, X. Jiang, and Z. Liu, "Strain effects in graphene and graphenenanoribbons: The underlying mechanism," Nano Res., vol. 3, pp. 545-556, Aug .2010

[11] H. Zhao, K. Min, and N. R. Aluru, "Size and chirality dependent elastic properties of graphenenanoribbons under uniaxial tension," Nano Lett., vol. 9, pp. 3012-3015. July 2009.

Rifky Syariati was born on 13 February 1989 in Cianjur, Indonesia. He received bachelor degree in physics in October 2011. Nowadays, He enrolled in the master program at Institut Teknologi Bandung, Indonesia. During at graduate student, he is a researcher and a lecturer assistant. His research fields are material computation. He also member of Physics of Electronic Materials Research Division, InstitutTeknologi Bandung. 